\title{
INDOOR LOCALIZATION IN SENSOR NETWORK WITH ESTIMATION OF DOA AND RSSI MEASUREMENT
}

\author{
Amrita A. Agashe ${ }^{1}$, Hemant Chavan ${ }^{2}$ \\ ${ }^{I}$ Associate Professor in Electronics Engineering Department, Walchand College of Engineering, Sangli, \\ amrita.agashe@walchandsangli.ac.in \\ ${ }^{2}$ M. Tech. Student in Electronics Engineering Department, Walchand College of Engineering, Sangli
}

\begin{abstract}
Due to advancement in MEMS technology today wireless sensor network has gained a lot of importance. The wide range of its applications includes environmental and habitat monitoring, object localization, target tracking, security surveillance etc. Wireless sensor networks consist of tiny sensor devices called as motes. The constrained computation power, battery power, storage capacity and communication bandwidth of the tiny motes pose challenging problems in the design and deployment of such systems. In this paper we provide study and prototype implementation of architecture and algorithm for network of tiny wireless sensors for localization purpose.
\end{abstract}

Key Words-sensor network; localization, RSSI; Angle-of-arrival (AoA); direction-of-arrival (DoA).

\section{INTRODUCTION}

A WSN is a network of devices that have sensing, actuation, processing, and wireless communication capability. Wireless sensor networks have several potential applications, especially in the area of military operations [1][2]. Sensors can be used to detect physical phenomena, such as motion, acceleration, acoustic and light. The collected and processed sensor data can be transmitted through wireless links. The main benefit of WSNs comes from the tiny size and low cost of the nodes, which makes it possible to deploy more number of nodes in a wide geographic area. For effective use of the data from the sensors, the position of the sensors must be known. Finding the location of the nodes manually is costly and sometimes it is not feasible. Therefore, nodes should estimate their own locations after deployment.

If both distance and angle measurements are available between pair of sensor nodes, the relative position between a pair of sensor nodes can be obtained, which makes the network localization more successful. The location of an arbitrary node with respect to a single beacon can be obtained by adding the relative position measurements between pairs of nodes (motes) lying in a path from the node to the beacon, and can be calculated in a distributed fashion [7]. To be suitable to WSNs, a device to measure relative angle between a receiver-transmitter must be tiny enough so that it can be integrated into a wireless sensor node. Specialized radio hardware equipment will not only increase the cost but also make it tedious to take advantage of continuous improvements in protocols for wireless sensor networking that is seen in past few years. The distance between two nodes by considering the fact that RSSI values calculated at the one node is inversely proportional to the square of distance between two nodes. In this paper, we test the practicability of a simple procedure for estimating the DoA (Direction of Arrival) of an incoming radio wave from a transmitter by using parabolic reflector at the receiver. When the reflector is coordinated in such a way that the incoming signal is limelight at the receiver antenna, a high RSSI value is observed. At other orientations of the reflector, a minimum RSSI is observed. The DoA can be estimated by choosing for the direction in which the maximum RSSI is observed.

\section{EXPERIMENTAL APPARATUS AND}

\section{METHOD}

The DoA measuring device, shown in Figure 1 consists of a Transceiver, rotational parabolic reflector, and a processor board. The transceiver is the Indian Institute of Technology, Hyderabad Mote ( IITH mote) with whip antenna, operating in the $2.4 \mathrm{GHz}$ band, which has the on board capability of measuring received signal strength. A 200 steps-per-revolution stepper motor was used to rotate the transceiver and reflector. The receiver is attached to the parabolic reflector in a way that the motor shaft and the antenna placed in the focal axis of the reflector. The parabolic reflector is a sheet of aluminum, bent into a parabolic form. Stepper motor is controlled by 8051 microcontroller. 


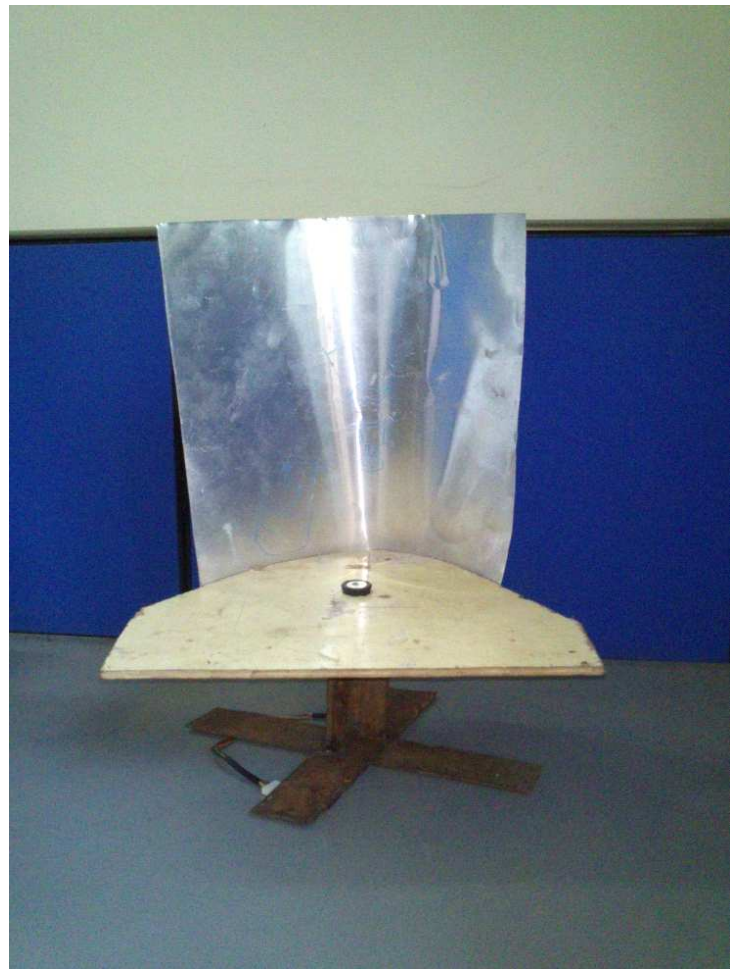

Fig-1: DoA measuring device

Figure 2 shows IITH mote, which is used in our experiments.

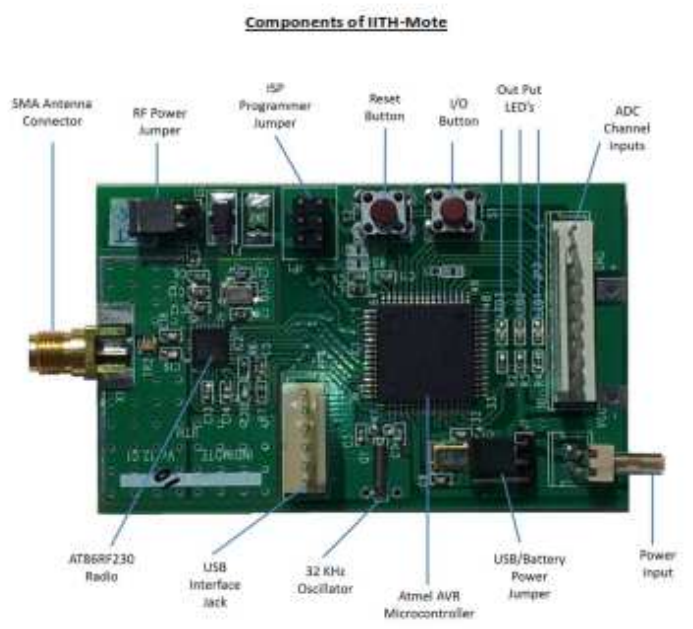

Fig-2: IITH mote

One full revolution of the stepper motor is taken in experiment for DoA and distance estimation between transmitter and receiver. Sender mote always sends the RSSI data to receiver. During experiments, RSSI values are received at every angular position of the reflector, which leads to a curve of RSSI versus angular position. The calculated DoA is the angular position of the reflector where the maximum signal strength has been achieved. Due to fluctuations in RSSI, the RSSI values are not directly used. The algorithm used to search for the maximum signal strength is described below.

\section{DOA SEARCH ALGORITHM}

At every single angular position of the reflector, we take at most 50 number of RSSI measurements within a time-out period of 3 seconds. Let $N_{j}$ be the number of measurements receives at single angular position $\theta=\theta_{j}$ of the reflector, where $j=1,2,3 \ldots . . .200$. With angular position $\theta_{j}=0,1.8, \ldots \ldots \ldots ., 358.2$ and $N_{j} \leq 50$ for every $j$. Let $r_{k}(j)$ be the value of $k^{\text {th }}$ RSSI measurement given at angular position $\theta_{j}$, where $\mathrm{k}=1,2,3 \ldots \ldots \ldots \ldots N_{j}$.

For IITH mote we have found that for our experiment RSSI value in between -36 to -100 (in $\mathrm{dbm}$ ). Let $\mathrm{S}$ denotes the matrix containing RSSI values at various angular positions. Elements of matrix $\mathrm{S}$ are calculated a $\mathrm{S}$

$$
S(i, j)=\frac{\text { no. of } k \text { that } \quad r_{k}(j)=-i d b m}{N_{j}}
$$

By observing the element of matrix $S$ we can get the angle between sender mote and receiver mote.

\section{DESIGN OF PARABOLIC REFLECTOR}

The equation of a parabola with focal length $F$ can be written in the $(\mathrm{x}, \mathrm{z})$ plane as:

$$
x^{2}=4 F(F-z) \cdot|x| \leq \frac{d}{2}
$$

The parabola is described by two parameters, the diameter D and the focal length $\mathrm{F}$. We also define two auxiliary parameters, the vertical height of the reflector $(\mathrm{H})$ and the max angle between the focal point and the edge of the dish $(\Theta)$. These parameters are related to each other by the following equations

$$
\begin{gathered}
\frac{F}{D}=\frac{1}{4 \tan \left(\frac{\theta}{2}\right)} . \\
F=\frac{D^{2}}{16 H} \ldots \ldots
\end{gathered}
$$

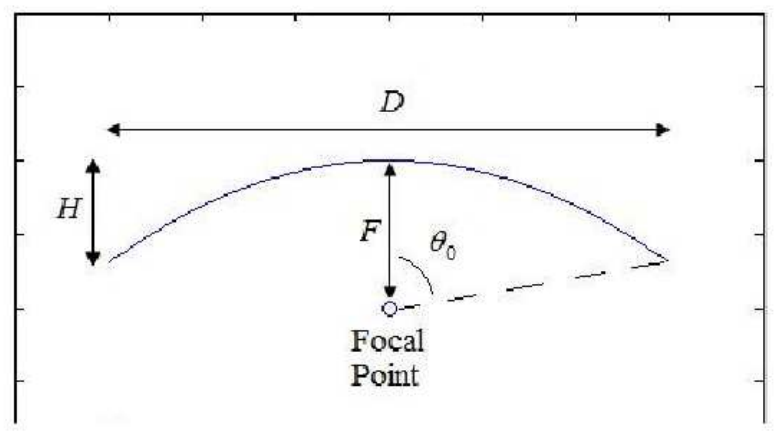

Fig-3: Focal point of parabolic Reflector 
To analyze the reflector, approximations from geometric optics are used. Since the reflector is large relative to a wavelength, this assumption is reasonable.

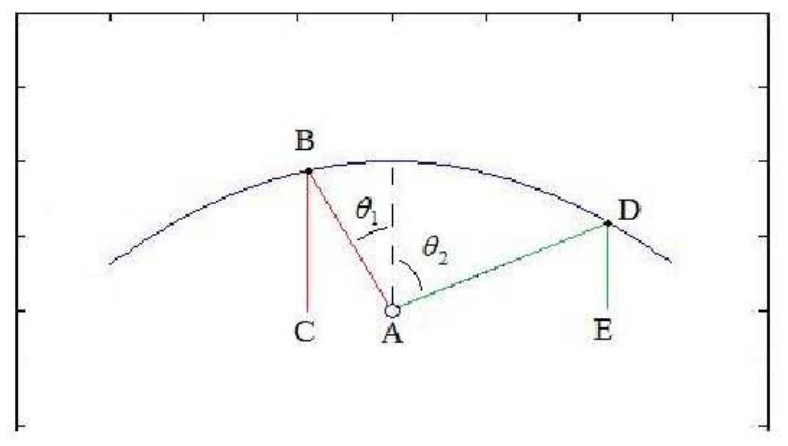

Fig-4 :Two rays leaving the focal point and reflected from the parabolic reflector

Analysis of the structure via straight line rays from the focal point is carried out with each ray acting as a plane wave. Consider two transmitted rays from the focal point, arriving from two distinct angles as shown in Figure4. The reflector is assumed to be perfectly conducting, so that the rays are completely reflected. It has been observed that both rays end up travelling in the downward direction and path angles ( $\angle \mathrm{ADE}$ and $<\mathrm{ABC}$ from Figure 4$)$ are equal.

All rays emanating from the focal point (the source or feed antenna) will be reflected towards the same direction. The distance each ray travels from the focal point to the reflector and then to the focal plane is constant. For design, the value of the diameter D should be increased to increase the gain of the antenna. The focal length $\mathrm{F}$ is the only free parameter; typical values are commonly given as the ratio F/D, which usually range between 0.3 and 1.0.

We have designed the parabolic reflector of the following specifications

$$
\begin{aligned}
& F=4 \mathrm{~cm} \\
& D=24 \mathrm{~cm} \\
& \mathrm{~h}=9 \mathrm{~cm} \\
& \mathrm{x}=76 \text { degree }
\end{aligned}
$$

\section{FINDING THE DISTANCE BETWEEN}

\section{TRANSMITTER AND RECEIVER}

By knowing the fact that RSSI value is inversely proportional to the square of distance between transmitter and receiver we get the distance between them.

Localization of sensor node:

With the calculation of the DoA and distance between sender and receiver we can get the accurate position of the sender with respect to receiver.

\section{EXPERIMENTAL RESULTS}

Figure 5 shows RSSI values in HEX at various angular positions. Further these RSSI values in HEX are converted into $\mathrm{dBm}$ which is shown in Figure 6.

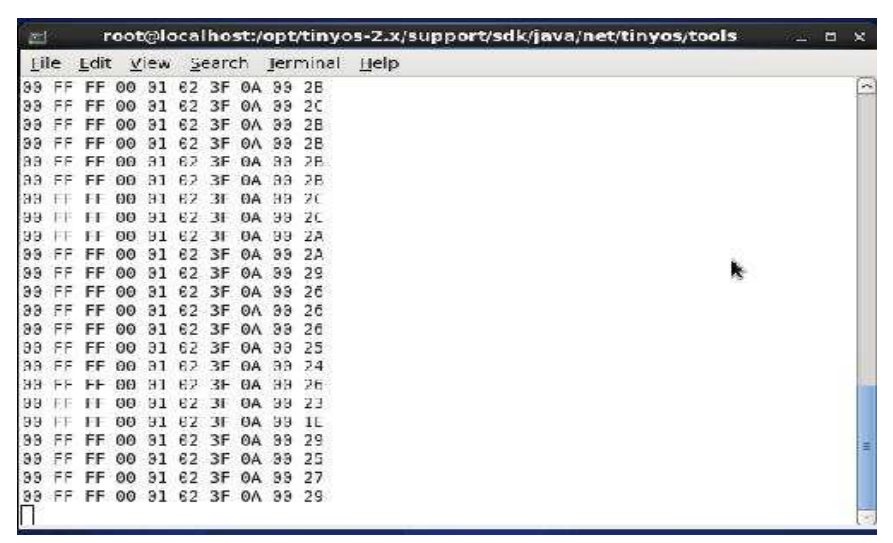

Fig-5: RSSI values in HEX at various angular positions

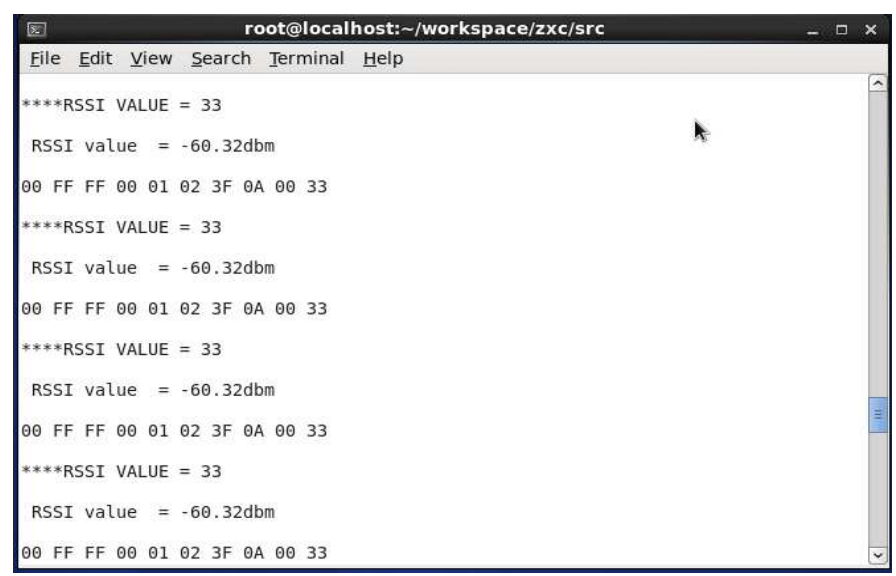

Fig-6: RSSI values in $\mathrm{dBm}$ at various angular positions

Serial forwarder is used to send data from PC to mote. The serial forwarder program opens a packet source and lets many applications connect to it over TCP/IP stream in order to use that source. Figure 7 shows our serial forwarder.

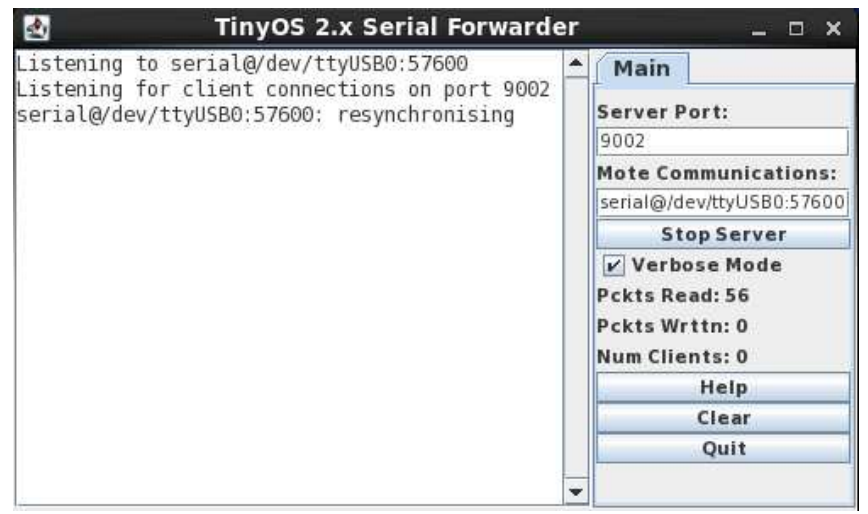

Fig-7: Serial Forwarder 


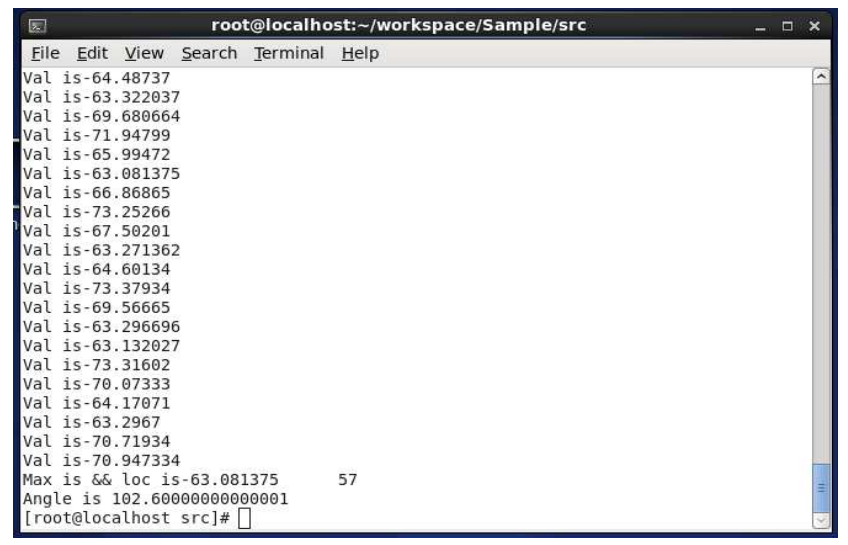

Fig-8: Angle between Transmitter and Receiver

Figure 8 shows the measured Angle between Transmitter and Receiver.

\begin{tabular}{|c|c|}
\hline RSSI in dbm & Distance in cm \\
\hline-78.56 & 400 \\
\hline-80.36 & 500 \\
\hline-82.36 & 600 \\
\hline-90.72 & 700 \\
\hline-91.00 & 800 \\
\hline-95.28 & 1000 \\
\hline-96.03 & 1100 \\
\hline
\end{tabular}

Table 1. RSSI and corresponding distance

Table 1 shows RSSI and corresponding distance between transmitting and receiving motes. By measuring the distance and angle between sensor nodes (motes) sensor node is localized.

\section{CONCLUSION}

The localization information is crucial for the operation of wireless sensor network. In this paper, we have located unknown node by incorporating RSSI and DoA information. The results of the experiment showed that it is possible to measure DoA and distance between motes with the help of RSSI and by using an actuated parabolic reflector, as long as there is less reflecting parameter in between transceivers.

\section{ACKNOWLEDGEMENT}

We are very much thankful to All India Council for Technical Education, New Delhi for sponsoring this work under Research Promotion Scheme (Ref. No. : 8023/RID/RPS-117/2011-12) 


\section{REFERENCES}

[1] L. Girod and D. Estrin, "Robust range estimation using acoustic and multimodal sensing," in Proc. IEEE/RSJ Int. Conf. Intell. Robot. Syst., Oct. 2001, pp. 1312-1320.

[2] S. Lanzisera, D. Lin, and K. Pister, "RF time of flight ranging for wireless sensor network localization," in Proc. Workshop on Intelligent Solutions in Embedded Systems (WISES), Jun. 2006, pp. 1-12.

[3] D. Moore, J. Leonard, D. Rus, and S. Teller, "Robust distributed network localization with noisy range measurements," in Proc. 2nd ACM Conf. Embedded Networked Sensor Syst., Nov. 2004, pp. 50-61.

[4] G. Mao, B. Fidan, and B. D. Anderson, "Wireless sensor network localization techniques," Comput. Networks vol. 51, no. 10, pp. 2529-2553, 2007. [Online]. Available: http://www.sciencedirect.com/science/article/ B6VRG4MR88Y3-1/2/e1ec9202368c395d70268cd7e44b6484

[5] A. Basu, J. Gao, J. Mitchell, and G. Sabhnani, "Distributed localizationby noisy distance and angle information," in Proc. 7th ACM Int. Symp. Mobile Ad Hoc Networking and Comput. (MobiHoc), May 2006, pp. 262273.

[6] K. Chintalapudi, A. Dhariwal, R. Govindan, and G. Sukhatme, "Ad-hoc localization using ranging and sectoring," in Proc. IEEE INFOCOMM, Marl 2004.

[7] P. Barooah, N. M. da Silva, and J. P. Hespanha, "Distributed optimalestimation from relative measurements for localization and time synchronization," in Distributed Computing in Sensor Systems DCOSS, ser. LNCS, P. B. Gibbons, T. Abdelzaher, J. Aspnes, and R. Rao, Eds. New York: Springer, 2006, vol. 4026, pp. 266-281. 